\title{
Periodontal Gel Dosage Form
}

National Cancer Institute

\section{Source}

National Cancer Institute. Periodontal Gel Dosage Form. NCI Thesaurus. Code C149750.

Semi-solid preparation consisting of a gel intended to be placed in the pouch between the tooth and the ging iva. 